\title{
High fracture toughness AIN achieved by addition of AIN whiskers and tape-casting
}

\author{
Hiroki SHIMIZU ${ }^{1,2}$, Naoki KONDO ${ }^{1, \dagger}$, Akihiro SHIMAMURA ${ }^{1}$, Mikinori HOTTA ${ }^{1}$, \\ Shunta HARADA ${ }^{2}$, Toru UJIHARA ${ }^{2}$ and Yoshihiro OHNISHI ${ }^{3}$ \\ ${ }^{1}$ National Institute of Advanced Industrial Science and Technology (AIST), \\ 2266-98 Anagahora, Shimo-Shidami, Moriyama-ku, Nagoya 463-8560, Japan \\ ${ }^{2}$ Nagoya University, Furo-cho, Chikusa-ku, Nagoya 464-8601, Japan \\ ${ }^{3}$ Furukawa Denshi Co., Ltd., Kodate 20, Kami-Yoshima, Yoshima-machi, Iwaki, Fukushima 970-1153, Japan
}

\begin{abstract}
Aluminum nitride (AIN) possesses excellent thermal conductivity and electrical resistivity, which makes it an ideal candidate for high-power, high-speed integrated circuit substrates. Its low fracture toughness requires the manufacturing of thicker substrates. However, this leads to degraded heat dissipation performance. Herein, we investigated a new strategy, combining the addition of AIN whiskers and tape-casting, to overcome the low fracture toughness disadvantage. The sintered AIN with AIN whiskers addition induced the formation of a highly anisotropic microstructure with aligned rod-like grains, effectively enhancing the fracture toughness to 6.7 MPa m ${ }^{1 / 2}$, while maintaining thermal conductivity but degrading strength.

(c)2022 The Ceramic Society of Japan. All rights reserved.
\end{abstract}

Key-words : Aluminum nitride, Whisker, Microstructure, Anisotropy, Toughness

[Received August 31, 2021; Accepted November 1, 2021]

Aluminum nitride (AIN) is used as an insulated heat dissipation substrate in several power devices due to its substantial thermal conductivity. ${ }^{1), 2)}$ However, its low fracture toughness necessitates the use of thicker substrates to ensure reliability. Thicker substrates exhibit higher thermal resistances, thus degrading the heat dissipation performance of integrated circuit substrates. Therefore, improving the fracture toughness of AlN can allow for the use of thinner substrates with enhanced heat dissipation, ensuring the realization of high-performance power devices.

Several approaches have been made for the toughening of AlN ceramics. One such approach was the use of sintering aids; however, an insufficient fracture toughness value was yielded $\left(\sim 3 \mathrm{MPa} \mathrm{m}^{1 / 2}\right){ }^{3)}$

Silicon nitride $(\mathrm{SN})$ is a ceramic with high fracture toughness, which is regarded as a reference in our approach to enhance the toughness properties of AIN. After sintering, $\mathrm{SN}$ grains tend to elongate via anisotropic grain growth and form rod-like grains. The rod-like grains exhibit a composite-like microstructure. Through the enhancement of toughening mechanisms owing to the microstructure, such as grain pull-out and crack deflection, high fracture toughness is finally obtained. ${ }^{4), 5)}$ Furthermore, by aligning the rod-like grains in the microstructure, the fracture toughness is expected to be significantly improved in a specific

\footnotetext{
Corresponding author: N. Kondo; E-mail: naoki-kondo@ aist.go.jp
}

direction. Microstructures with such features are created by tape-casting combined with seed particle addition. ${ }^{6), 7)}$

Anisotropic grain growth, and by extension the development of rod-like grains, is difficult in AlN. ${ }^{8)}$ Therefore, the addition of rod-like grains in the microstructure of AIN constitutes an approach worth considering. In a recent study, researchers successfully designed AlN whiskers both in the laboratory, ${ }^{9)}{ }^{10)}$ as well as in the commercial environment.

In this work, considering the toughening model of $\mathrm{SN}$ as a reference, ${ }^{6)}$ we intended to make a microstructure of AIN with aligned rod-like grains by employing a tape-casting technique combined with AIN whiskers addition. Then, fracture toughness of the AlN was evaluated, and the possibility of our approach, AlN whiskers addition and tape casting, was confirmed.

AlN powder (particle size $0.4 \mu \mathrm{m}, \mathrm{H}$ Grade, Tokuyama Corp., Tokyo, Japan) and AIN whiskers (U-map Co., LTD. Aichi, Japan) were used as the raw materials. The AlN whiskers were mechanically shortened by using a ball mill, and their shapes were analyzed by the PITA-04 system (SEISHIN ENTERPRISE Co., LTD. Tokyo, Japan). In PITA-04, the AIN whiskers were dispersed in ethanol and flowed in a special cell (chip), where a high-speed and high-pixel density CCD camera captured their images. Subsequently, the shape parameters of individual AIN whiskers, diameter (D), length (L), and aspect ratio (A), were measured from the photographs. Yttria $\left(\mathrm{Y}_{2} \mathrm{O}_{3}\right)$ was 
chosen as the sintering additive because it can facilitate the achievement of high thermal conductivity. ${ }^{3), 11)}$

Fabrication of a sintered sample was conducted referring to a previous report. ${ }^{6)}$ AlN powder, AlN whiskers, and $\mathrm{Y}_{2} \mathrm{O}_{3}$ powder were added into a mixed solvent, composing of an organic solvent, a dispersant, an organic binder, and a plasticizer. The weight ratio of the AlN powder, AIN whiskers, and $\mathrm{Y}_{2} \mathrm{O}_{3}$ powder was 100:3:5, respectively. Next, the solvent was ball-milled to make a slurry. Green sheets of $150 \mu \mathrm{m}$ in thickness were then fabricated from the slurry via tape-casting. The sheet was cut and stacked, then, the stacked sheets were thermally compression bonded at $150^{\circ} \mathrm{C}$ for $30 \mathrm{~min}$ under a pressure of $20 \mathrm{MPa}$ to obtain a green body with dimensions of $50 \times 45 \times 6 \mathrm{~mm}$. The green body was calcined, and then sintered at $1900^{\circ} \mathrm{C}$ for $2 \mathrm{~h}$ using a graphite resistance furnace in a nitrogen atmosphere. The directions of the obtained sintered body were denoted as follows: machine (sheet feed) direction: MD, transverse to machine direction: TD, and normal direction in sheet plane: ND. The planes normal to each direction were M plane: MP, T plane: TP, and N plane: NP, respectively. These directions and planes are illustrated in Fig. 1. For comparison, a sintered AlN body without AIN whiskers addition was also fabricated in a similar way. Samples for evaluation were cut from the sintered bodies.

The crystalline phases and anisotropy of the sample were analyzed via X-ray diffraction (XRD). Microstructure the sample was observed by scanning electron microscopy (SEM). Fractured surfaces of the sample were provided for SEM observation. The thermal conductivity was measured using a laser flash method, through which ND was the heat flow direction. The strength was measured by the fourpoint bending test following ISO 14704 (sample size: $4.0 \mathrm{~mm}$ thickness $\times 3.0 \mathrm{~mm}$ width $\times 50 \mathrm{~mm}$ length, outer

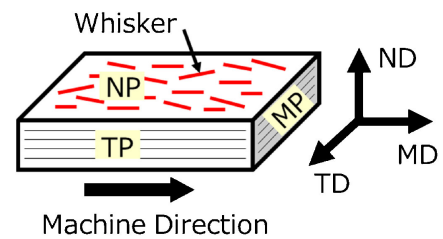

Fig. 1. Schematic drawing showing the directions and planes of the sintered body. and inner span: 10 and $30 \mathrm{~mm}$ ). The fracture toughness was measured using the single edge pre-cracked beam (SEPB) method following ISO 15732 (sample size: 3.0 $\mathrm{mm}$ thickness $\times 4.0 \mathrm{~mm}$ width $\times 20 \mathrm{~mm}$ length, span: 16 $\mathrm{mm})$. The crack propagation direction and crack plane were ND and MP, respectively.

An SEM image of the AlN whiskers along with the diameter and length distributions revealed by image analysis are illustrated in Fig. 2. The rod-like shape of the AlN whiskers, which had grown along the $c$-axis, ${ }^{9), 10)}$ was evident. Their shape parameters are shown in Table 1. Almost all AlN whiskers possessed larger diameters compared with the AlN powder particles. Some finer particles and fragments of whiskers were also found. These seemed to be generated during the whisker synthesis and mechanically shortening procedure.

Properties of the sintered samples with and without AlN whiskers addition are shown in Table 2. The samples with and without whiskers exhibited densities of 3.33 and 3.30 $\mathrm{g} \mathrm{cm}^{-3}$, respectively, and thermal conductivities of 178 and $184 \mathrm{~W} \mathrm{~m}^{-1} \mathrm{~K}^{-1}$, respectively. The insignificant differences between these values suggested that the AIN whiskers addition did not affect the sintering performance.

The XRD patterns of the MP, TP, and NP of the sample with AlN whiskers are shown in Fig. 3 together with the

Table 1. Cumulative diameter, length, and aspect ratio at 10, 50, and 90 vol $\%$ of AlN whiskers

\begin{tabular}{lccc}
\hline & \multicolumn{3}{c}{ Cumulative volume $(\%)$} \\
\cline { 2 - 4 } & 10 & 50 & 90 \\
\hline Diameter $(\mu \mathrm{m})$ & 0.9 & 1.8 & 3.7 \\
Length $(\mu \mathrm{m})$ & 7.8 & 16.9 & 44.4 \\
Aspect ratio & 1.7 & 5.2 & 20.1 \\
\hline
\end{tabular}

Table 2. Properties of sintered AIN with and without AlN whiskers addition

\begin{tabular}{|c|c|c|c|c|}
\hline & $\begin{array}{l}\text { Density, } \\
\left(\mathrm{g} \mathrm{cm}^{-3}\right)\end{array}$ & $\begin{array}{c}\text { Thermal } \\
\text { conductivity, } \\
\left(\mathrm{W} \mathrm{m}^{-1} \mathrm{~K}^{-1}\right)\end{array}$ & $\begin{array}{c}\text { Flexural } \\
\text { strength, } \\
(\mathrm{MPa})\end{array}$ & $\begin{array}{c}\text { Fracture } \\
\text { toughness, } \\
\left(\mathrm{MPa} \mathrm{m}^{1 / 2}\right)\end{array}$ \\
\hline with AlN-whiskers addition & 3.33 & 178 & 318 & 6.7 \\
\hline $\begin{array}{l}\text { without AlN-whiskers } \\
\text { addition }\end{array}$ & 3.30 & 184 & 438 & 3.1 \\
\hline
\end{tabular}

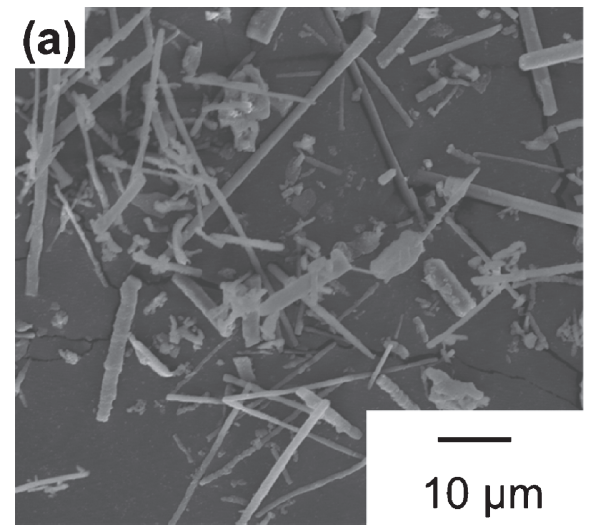

(b)

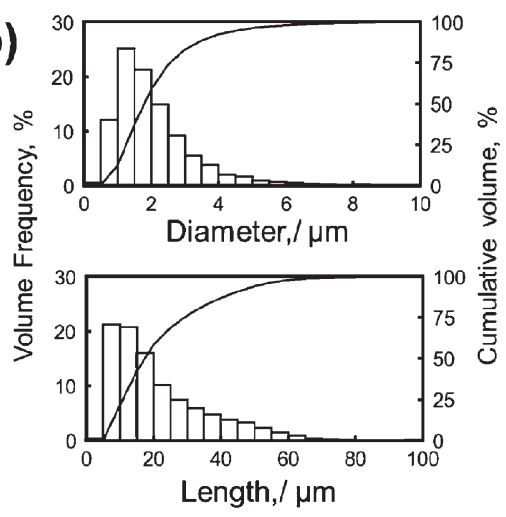

Fig. 2. (a) SEM image of AlN whiskers; (b) Diameter and length distributions of AlN whiskers. 
JCPDS 25-1133 pattern, which corresponded to the theoretical pattern of the isotropic AlN. The (1011) and (0002) diffraction peaks from the NP were lower compared with the corresponding peaks from the isotropic pattern. The peaks from the TP also demonstrated a similar tendency. The (1010) and (0002) peak height ratios from the XRD patterns of the MP, TP, and NP were calculated for quantitative evaluation, and determined to be $1.31,9.87$, and 29.7 , respectively. These results indicated the strong alignment of the AlN whiskers along the MD direction and the formation of an anisotropic microstructure. Additionally, $\mathrm{XRD}$ analysis revealed the secondary crystalline phase of the sintered sample to be $2\left(\mathrm{Y}_{2} \mathrm{O}_{3}\right) \cdot 3\left(\mathrm{Al}_{2} \mathrm{O}_{3}\right)$ (yttrium aluminum perovskite, YAP), which is frequently detected in AlN microstructures with $\mathrm{Y}_{2} \mathrm{O}_{3}$ additives. ${ }^{12)}$ Therefore, the detection of YAP was reasonable.

The SEM images of the fractured surfaces of the NP and MP of the sample with AIN whiskers addition are shown in Figs. 4(a) and 4(b), together with the NP of that without AlN whiskers addition in Fig. 4(c). The fractured surface of the MP of the sample without AIN whiskers addition exhibited identical morphologies to the NP, Fig. 4(c).

Coarse grains with a rod-like shape occupied approximately $60 \%$ of the NP from the sample with AIN whiskers

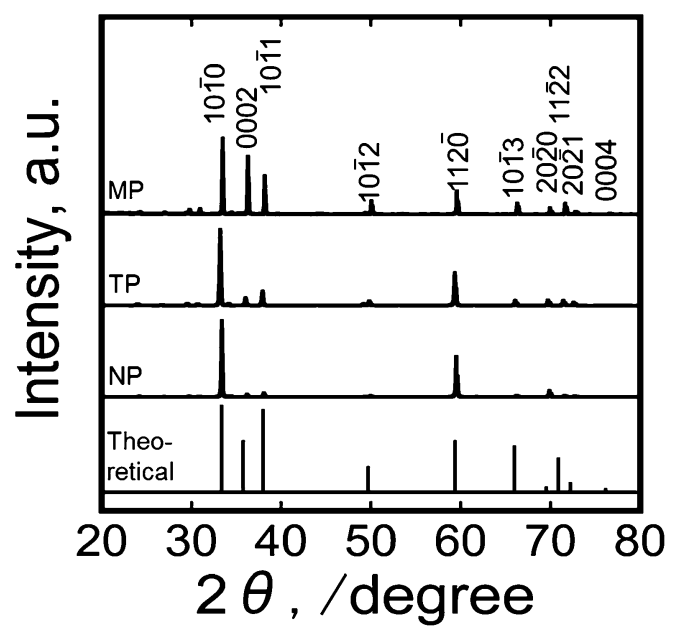

Fig. 3. XRD patterns of the sample with AlN whiskers addition across the MP, TP, and NP. The theoretical isotropic pattern calculated for AlN can be seen at the bottom. addition, which was aligned along the MD direction. Some of the coarse grains in the MP exhibited hexagonal crosssections, originating from the fractured c-plane of the AlN crystal. In AlN ceramics added with coarse AlN grains ${ }^{13)}$ or AlN whiskers, ${ }^{14)}$ pores exceeding $10 \mu \mathrm{m}$ were sometimes observed around the coarse grains or whiskers. However, similar pores were rarely observed in this work.

Finer grains were also observed among the coarser grains, with sizes comparable to those observed in the sample without AIN whiskers addition. It was therefore deduced that the coarser and finer grains originated from the AlN whiskers and raw powder particles, respectively. The AlN whiskers addition and the tape-casting process resulted in the formation of aligned rod-like grains in the microstructure of the samples. Finer particles and fragments of whiskers were contained in the added whiskers. Their sizes were smaller and amounts were less compared to the whiskers, thus, it is considered that they had little effect on the microstructure formation.

Flexural strength and fracture toughness of the samples with and without AlN whiskers addition are shown in Table 2. Fracture toughness was improved from 3.1 to 6.7 $\mathrm{MPa} \mathrm{m}^{1 / 2}$, but flexural strength degraded from 431 to 318 $\mathrm{MPa}$, by AlN whiskers addition. Therefore, the addition and alignment of AlN whiskers in the sintered AIN greatly enhanced the fracture toughness, but degraded strength.

The improved fracture toughness should be due to the fracture mode change caused by the AlN whiskers addition. Intergranular fracture was the major fracture mode in the sample without AlN whiskers addition, whereas transgranular fracture was that in the sample with AlN whiskers addition. To validate the fracture mode, tilted fracture surfaces were observed by SEM. The results are shown in Fig. 5. Some fractured coarse rod-like grains, protruded more than $10 \mu \mathrm{m}$, were found in the sample with AlN whiskers addition [Fig. 5(a)]. This was the evidence of the crack deflection, which led to toughening. The AlN whiskers addition, however, increased the number of larger grains which acted as defects, resulted in degraded strength. On the contrary, in the sample without AlN whiskers addition, fracture occurred at grain boundaries, and crack deflection was not so remarkable [Fig. 5(b)]. This was the reason for low fracture toughness.
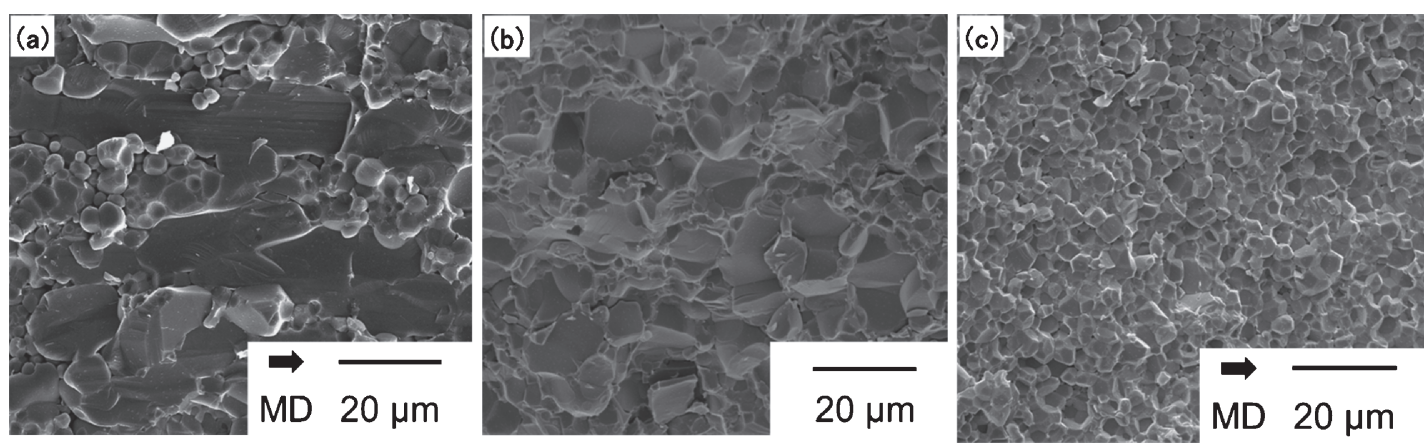

Fig. 4. SEM images of the fractured surfaces of the samples: (a) NP and (b) MP of the sample with AIN whiskers addition; (c) NP of the sample without AIN whiskers addition. (b) was taken from the fractured SEPB specimen, and crack propagation direction was from bottom to top. 

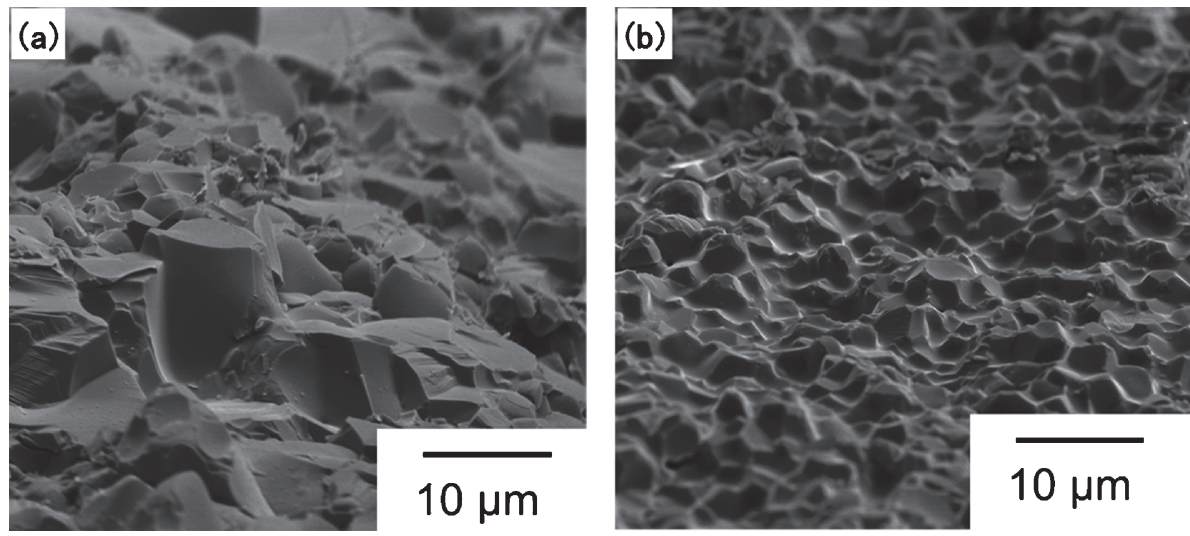

Fig. 5. SEM images of the fractured MP: (a) with and (b) without AIN whiskers addition. The images were taken from $75^{\circ}$ tilted planes. Crack propagation direction was from bottom to top.

In conclusion, high fracture toughness AIN was successfully fabricated by the AlN whiskers addition and tape-casting. The sintered AlN sample with aligned AlN rod-like grains exhibited a fracture toughness of 6.7 $\mathrm{MPa} \mathrm{m}^{1 / 2}$, which was approximately twice that of the sample without AIN whiskers addition. This high fracture toughness was achieved by the enhanced toughening mechanisms, crack deflection, owing to its microstructure with aligned rod-like grains.

Acknowledgements This paper is based on the results of a project, JPNP12004, subsidized by the New Energy and Industrial Technology Development Organization (NEDO), Japan.

\section{References}

1) G. A. Slack, J. Phys. Chem. Solids, 34, 321-335 (1973).

2) A. K. Knudsen, Am. Ceram. Soc. Bull., 97, 32-38 (2018).

3) R. Terao, J. Tatami, T. Meguro and K. Komeya, J. Eur. Ceram. Soc., 22, 1051-1059 (2002).

4) K. Hirao, T. Nagaoka, M. E. Brito and S. Kanzaki, J. Am. Ceram. Soc., 77, 1857-1862 (1994).

5) P. F. Becher, E. Y. Sun, K. P. Plucknett, K. B. Alexander, C.-H. Hsueh, H.-T. Lin, S. B. Waters,
C. G. Westmoreland, E.-S. Kang, K. Hirao and M. E. Brito, J. Am. Ceram. Soc., 81, 2821-2830 (1998).

6) K. Hirao, M. Ohashi, M. E. Brito and S. Kanzaki, J. Am. Ceram. Soc., 78, 1687-1690 (1995).

7) S. Kanzaki, M. E. Brito, M. C. Valecillos, K. Hirao and M. Toriyama, J. Eur. Ceram. Soc., 17, 1841-1847 (1997).

8) M. Tajika, H. Matsubara and W. Rafaniello, J. Ceram. Soc. Jpn., 105, 928-933 (1997).

9) R. Kobayashi, Y. Fukutomi and T. Takagi, J. Ceram. Soc. Jpn., 124, 1161-1163 (2016).

10) M. Matsumoto, H. Saitou, Y. Takeuchi, S. Harada, M. Tagawa and T. Ujihara, J. Cryst. Growth, 468, 576-580 (2017).

11) T. B. Jackson, A. V. Virkar, K. L. More, R. B. Dinwiddie, Jr. and R. A. Cutler, J. Am. Ceram. Soc., 80, 1421-1435 (1997).

12) D. Huang, Z. Tian, W. Cui, L. Gao, Z. Liu, X. Diao and G. Liu, Ceram. Int., 44, 20556-20559 (2018).

13) H. Nakata, J. Tatami, M. Namiki, K. Komeya, T. Meguro, A. Kuibira, M. Naito and T. Hotta, J. Ceram. Soc. Jpn., 112, 35-39 (2004) [in Japanese].

14) H. Okazaki, R. Kobayashi, R. Hashimoto, E. Fukushi and J. Tatami, J. Ceram. Soc. Jpn., 128, 991-994 (2020). 